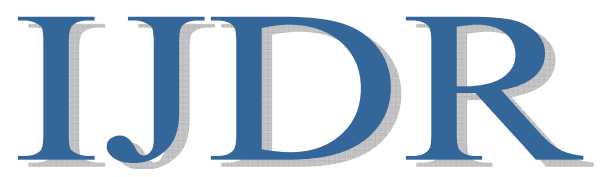

International Journal of Development Research

Vol. 11, Issue, 05, pp. 47329-47332, May, 2021

https://doi.org/10.37118/ijdr.21984.05.2021

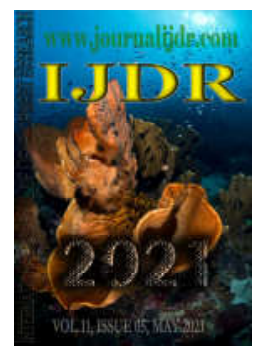

\title{
RENDIMIENTO Y EFICIENCIA EN EL USO DEL AGUA DE UN GENOTIPO DE CHILE SERRANO (Capsicum annuum L.) PRODUCIDO BAJO FERTILIZACIÓN ORGÁNICA E INORGÁNICA EN CASA SOMBRA
}

\section{*1José Jiménez León, 2Jesús López Elías, ${ }^{3}$ Marco A. Huez López and ${ }^{4}$ Alfonso Sánchez Villegas}

${ }^{1}$ Doctorado en Ciencias Agropecuarias por la Universidad Autónoma de Baja California, México; ${ }^{2}$ Doctorado en Producción Vegetal por la Universidad de Almería, España; ${ }^{3}$ Doctor en Filosofía (PhD) por New México State University, USA; ${ }^{4}$ Maestría en Ciencias en Horticultura por la Universidad de Sonora, México.

\section{ARTICLE INFO}

\section{Article History:}

Received $08^{\text {th }}$ February, 2021

Received in revised form

$26^{\text {th }}$ March, 2021

Accepted $11^{\text {th }}$ April, 2021

Published online $30^{\text {th }}$ May, 2021

\section{Key Words:}

Fertilization,

Organic agriculture

Green pepper.

\begin{abstract}
The objective of this research was to evaluate the effect of organic fertilization, compared to inorganic and a combination of both in the cultivation of serrano pepper (Capsicum annuum L.) under shade net at the University of Sonora. The variables that were evaluated were: fruit weight, yield, length and diameter of fruit, as well as efficiency of water use. For the weight of the fruit per plant, no significant differences were observed between treatments in the first two cuts, however, for the third cut if there were significant differences, the best treatments were inorganic fertilization and the mixture of inorganic plus algae extracts with 280.29 and $212.21 \mathrm{~g}$ respectively. For the fourth and fifth cut the trend was in the same way up to the final average resulting in the best treatment inorganic fertilization with $233.94 \mathrm{~g}$ per plant followed by the mixture of inorganic plus seaweed extract with $166.75 \mathrm{~g}$ per plant. Regarding the yield, it can be observed that from the third cut the treatments that contained inorganic resulted with the highest yield, resulting in the total average inorganic fertilization with $38665.77 \mathrm{~kg} \mathrm{ha}^{-1}$ and inorganic fertilization plus algae extract with $27514.08 \mathrm{~kg} \mathrm{ha}^{-1}$. The results obtained from this research show that organic fertilization does not provide high yields compared to inorganic because of the gradual release of nutrients to the plant.
\end{abstract}

Copyright (C) 2021, José Jiménez León et al. This is an open access article distributed under the Creative Commons Attribution License, which permits unrestricted use, distribution, and reproduction in any medium, provided the original work is properly cited.

Citation: José Jiménez León, Jesús López Elías, Marco A. Huez López and Alfonso Sánchez, Villegas, 2021. "Rendimiento y eficiencia en el uso del agua de un genotipo de chile serrano (Capsicum annuum L.) producido bajo fertilización orgánica e inorgánica en casa sombra", International Journal of Development Research, 11, (05), 47329-47332.

\section{INTRODUCIÓN}

En la última década la superficie sembrada en México con chile registró una tasa de crecimiento media anual de $0.6 \%$; el rendimiento aumentó y la producción mantuvo un ritmo de crecimiento de $1.5 \%$. Este comportamiento es el resultado de la incorporación de avanzados sistemas de producción, incluyendo la agricultura protegida (INFORURAL, 2012). Los estados que concentran más del $50 \%$ de la superficie plantada y cosechada, así como el $60 \%$ de la producción, son los siguientes: Sinaloa, Chihuahua, Guanajuato, Sonora y Zacatecas. La mayor parte de la producción de chile mexicano destinado a la exportación proviene de la costa noroeste, particularmente los estados de Sinaloa y Sonora, lo que le permite ser uno de los principales abastecedores de chile en los mercados de Estados Unidos, Canadá, Japón y Alemania, principalmente en ciclo invierno-primavera (Partida-Sandoval y Quezada-Camberos, 2012). En la actualidad, una de las principales tareas de los científicos es encontrar formas naturales de mejorar la productividad de las plantas, que conduzca a una agricultura respetuosa con el medio ambiente mejorar la sostenibilidad de los sistemas agrícolas (Du Jardín, 2015; Van Oosten et al., 2017). Los abonos orgánicos constituyen una opción sostenible, aunque no contengan los nutrimentos suficientes para la obtención de cosechas de alto rendimiento, porque promueven el incremento de la materia orgánica del suelo, la actividad microbiana y una liberación gradual de nutrientes a la planta, para una nutrición mineral más balanceada (Nicholls y Altieri, 2006). El uso de elevadas dosis de fertilizantes sintéticos, nitrogenados y fosfatados, representa un alto costo y causa graves daños al ambiente (Montaño et al., 2009). La agricultura ecológica es una alternativa que permite, además de sustituir los insumos tradicionales, mantener y mejorar la calidad del suelo, la producción de alimentos libre de trazabilidad no sostenible, dado que no incorpora en los sistemas de producción, agroquímicos que afectan los recursos agua, suelo y medio ambiente (Rodríguez-Dimas et al., 2007). En la actualidad existe gran preferencia entre los consumidores por los alimentos frescos, libres de agroquímicos, inocuos y con alto valor nutricional; una alternativa para su generación es la producción orgánica, sistema que prohíbe el uso de productos sintéticos (Trewavas, 2004). El uso indiscriminado de fertilizantes químicos en la agricultura, aunque 
puede potenciar el rendimiento de las hortalizas y otros cultivos, también ha contribuido a un empobrecimiento de las características biológicas del suelo y daños al medio ambiente. Por el contrario, se ha demostrado que la aplicación de fertilizantes orgánicos posee ventajas, ya que se puede lograr la fertilidad química, física y biológica del suelo con un menor impacto sobre el medio ambiente. Por otra parte, los costos de las aplicaciones de los fertilizantes orgánicos por hectárea son menores en comparación con los productos minerales de síntesis (Rai et al., 2014). Por ello el objetivo de esta investigación, fue evaluar el efecto de la fertilización orgánica, comparada con la inorgánica y una combinación de ambas en el cultivo de chile serrano (Capsicum annuum L.) Hib.Don Vicente, bajo casa sombra. Pretendiendo obtener producciones que resulten sostenibles y rentables para el agricultor y a la vez contribuyan a la seguridad alimentaria.

\section{MATERIALES Y MÉTODOS}

La presente investigación se realizó bajo casa sombra durante el ciclo primavera-verano de 2019, en el Campo Experimental del Departamento de Agricultura y Ganadería de la Universidad de Sonora $\left(29^{\circ} 00^{\prime} 51^{\prime \prime}\right.$ latitud norte, $111^{\circ} 07^{\prime} 59^{\prime \prime}$ longitud oeste y una altitud de 149 MSNM), clima BW(h) hw(e) el cual corresponde a la categoría de muy árido, extremoso y cálido (García, 1988). Se utilizó un área de casa sombra de $375 \mathrm{~m}^{2}$. Se evaluó un genotipo de chile Serrano (Capsicum annuum L) híbrido Don Vicente. La densidad de plantación fue de 3.3 plantas $\mathrm{m}^{-2}$., la aplicación del riego se realizó cuando la tensión de humedad alcanzó valores de -15 a $-20 \mathrm{kPa}$ en tensiómetros colocados a $30 \mathrm{~cm}$ de profundidad, usando cinta colocada superficialmente al lado de la hilera del cultivo, con goteros separados $30 \mathrm{~cm}$ y gasto de $1 \mathrm{~L} \mathrm{~h}^{-1}$. Los tratamientos evaluados fueron Lixiviado de lombriz $300 \mathrm{~L} \mathrm{ha}^{-1}$, Composta Gallinaza $9 \mathrm{t} \mathrm{ha}^{-1}$, Lombricomposta $5 \mathrm{t} \mathrm{ha}^{-1}$, fertilización inorgánica $290 \mathrm{~N}-75 \mathrm{P}-250 \mathrm{~K}-$ $70 \mathrm{Ca}-45 \mathrm{Mg} \mathrm{kg} \mathrm{ha}{ }^{-1}$ y Fertilización inorgánica + Extracto de algas $2 \mathrm{~L}$ $\mathrm{ha}^{-1}$. Las variables que se evaluaron fueron: peso del fruto, rendimiento, longitud y diámetro del fruto, al igual que la Eficiencia del Uso del Agua.
El experimento se realizó de acuerdo a un diseño completamente al azar, con cinco tratamientos y cuatro repeticiones. Los datos de cada variable de estudio fueron procesados mediante análisis de varianza (ANOVA) y comparación de medias de Tukey $(P \leq 0.05)$, usando el programa estadístico SAS (SAS Institute Inc., 2006).

\section{RESULTADOS Y DISCUSIÓN}

El periodo de producción de chile Serrano fue de 60 días, realizándose el primer corte a los 85 días y acumulando cinco cortes en total.

Peso del fruto: Para el peso del fruto por planta (Tabla 1) No se observaron diferencias significativas entre tratamientos en los primeros dos cortes, sin embargo para el tercer corte si hubo diferencias significativas, resultando los mejores tratamientos la fertilización inorgánica y la mezcla de inorgánica más extractos de algas con 280.29 y 212.21 g respectivamente. Para el cuarto y quinto corte la tendencia fue de lamisma manera hasta el promedio final resultando el mejor tratamiento la fertilización inorgánica con 233.94 g por planta seguido de la mezcla de inorgánico más extracto de algas con 166.75 g por planta. Los resultados obtenidos coinciden con De la Cruz-Lázaro et al. (2010), quienes observaron en cultivo de chile piquín la diferencia en el rendimiento obtenido con respecto a las fuentes de fertilización, encontraron mayor rendimiento en los sistemas de producción inorgánica.

Rendimiento: Respecto al rendimiento (Tabla 2) se puede observar que los tratamientos orgánicos en el primer corte resultaron mayores que el inorgánico y la mezcla con algas, aunque no hubo diferencias significativas. Para el segundo corte tampoco hubo diferencias significativas, pero a partir del tercer corte se resultaron con mayor rendimiento los tratamientos que contenían inorgánicos resultando el promedio total la fertilización inorgánica con $38665.77 \mathrm{~kg} \mathrm{ha}^{-1}$ y la fertilización inorgánica más extracto de algas con $27514.08 \mathrm{~kg} \mathrm{ha}^{-1}$.

Tabla 1. Peso individual de frutos (g) por planta de chile Serrano (Capsicum annuum L.) var. Don Vicente producido bajo fertilización orgánica e inorgánica, en casa sombra

\begin{tabular}{lcccccc}
\hline Tratamiento & Corte I & Corte II & Corte III & Corte IV & Corte V & Total \\
\hline Lixiviado & $77.35 \mathrm{a}^{\mathrm{a}}$ & $112.00 \mathrm{a}$ & $64.94 \mathrm{~b}$ & $83.49 \mathrm{~b}$ & $60.41 \mathrm{~b}$ & 79.64 \\
Gallinaza & $81.91 \mathrm{a}$ & $110.88 \mathrm{a}$ & $103.08 \mathrm{~b}$ & $148.75 \mathrm{~b}$ & $86.03 \mathrm{~b}$ & 106.13 \\
Vermicomposta & $69.46 \mathrm{a}$ & $78.00 \mathrm{a}$ & $105.81 \mathrm{~b}$ & $108.50 \mathrm{~b}$ & $168.70 \mathrm{ab}$ & 106.09 \\
Fert. Inorgánica & $45.90 \mathrm{a}$ & $86.50 \mathrm{a}$ & $280.29 \mathrm{a}$ & $436.50 \mathrm{a}$ & $320.50 \mathrm{a}$ & 233.94 \\
Inorg.+Orgânica & $43.05 \mathrm{a}$ & $99.50 \mathrm{a}$ & $212.21 \mathrm{a}$ & $320.00 \mathrm{ab}$ & $159.00 \mathrm{ab}$ & 166.75 \\
\hline
\end{tabular}

${ }^{\natural}$ Medias con la misma letras no son significativamente diferentes (Tukey $\mathrm{p} \leq 0.05$ ).

Tabla 2. Rendimiento $\left(\mathrm{kg} \mathrm{ha}^{-1}\right)$ de chile serrano (Capsicum annuum L.) var. Don Vicente producido bajo fertilización orgánica e inorgánica, en casa sombra

\begin{tabular}{lcccccc}
\hline Tratamiento & Corte I & Corte II & Corte III & Corte IV & Corte V \\
\hline Lixiviado & $2552.5 \mathrm{a}$ & $3696 \mathrm{a}$ & $2143.02 \mathrm{~b}$ & $2755.17 \mathrm{~b}$ & $1993.53 \mathrm{~b}$ & 13140.22 \\
Gallinaza & $2703.03 \mathrm{a}$ & $3659 \mathrm{a}$ & $3401.64 \mathrm{~b}$ & $4908.75 \mathrm{~b}$ & $2838.99 \mathrm{~b}$ & 17511.41 \\
Vermicomposta & $2292.18 \mathrm{a}$ & $2574 \mathrm{a}$ & $3491.73 \mathrm{~b}$ & $3580.5 \mathrm{~b}$ & $5567.1 \mathrm{ab}$ & 17505.51 \\
Fert. Inorgánica & $1514.70 \mathrm{a}$ & $2920.5 \mathrm{a}$ & $9249.57 \mathrm{a}$ & $14404.5 \mathrm{a}$ & $10576.5 \mathrm{a}$ & 38665.77 \\
Inorg.+Orgânica & $1420.65 \mathrm{a}$ & $3283.5 \mathrm{a}$ & $7002.93 \mathrm{a}$ & $10560 \mathrm{ab}$ & $5247 \mathrm{ab}$ & 27514.08 \\
\hline
\end{tabular}

${ }^{\top}$ Medias con la misma letras no son significativamente diferentes (Tukey $\mathrm{p} \leq 0.05$ ).

Tabla 3. Longitud (cm) por fruto de chile Serrano (Capsicum annuum L.) var. Don Vicente producido bajo fertilización orgánica e inorgánica, en casa sombra

\begin{tabular}{lcccccc}
\hline Tratamiento & Corte & Corte II & Corte III & Corte IV & Corte V & Total \\
\hline Lixiviado & $8.75 \mathrm{a}^{\mathbb{9}}$ & $9.33 \mathrm{ab}$ & $8.65 \mathrm{a}$ & $8.13 \mathrm{a}$ & $7.75 \mathrm{a}$ & 8.52 \\
Gallinaza & $8.32 \mathrm{a}$ & $9.00 \mathrm{ab}$ & $8.87 \mathrm{a}$ & $8.25 \mathrm{a}$ & $7.50 \mathrm{a}$ & 8.39 \\
Vermicomposta & $8.41 \mathrm{a}$ & $8.75 \mathrm{~b}$ & $8.83 \mathrm{a}$ & $8.25 \mathrm{a}$ & $7.98 \mathrm{a}$ & 8.44 \\
Fert. Inorgánica & $8.49 \mathrm{a}$ & $9.45 \mathrm{a}$ & $8.95 \mathrm{a}$ & $8.95 \mathrm{a}$ & $8.36 \mathrm{a}$ & 8.84 \\
Inorg.+Orgánica & $8.10 \mathrm{a}$ & $7.75 \mathrm{c}$ & $8.88 \mathrm{a}$ & $8.23 \mathrm{a}$ & $7.98 \mathrm{a}$ & 8.19 \\
\hline
\end{tabular}

${ }^{\natural}$ Medias con la misma letras no son significativamente diferentes (Tukey $p \leq 0.05$ ). 
Tabla 4. Diámetro (cm) por fruto de chile serrano (Capsicum annuum L.) var. Don Vicente producido bajo fertilización orgánica e inorgánica, en casa sombra

\begin{tabular}{|c|c|c|c|c|c|c|}
\hline Tratamiento & Corte I & Corte II & Corte III & Corte IV & Corte V & Total \\
\hline Lixiviado & $1.26 b^{9}$ & $1.46 \mathrm{a}$ & $1.49 \mathrm{a}$ & $1.49 \mathrm{a}$ & $1.52 \mathrm{a}$ & 1.44 \\
\hline Gallinaza & $1.26 \mathrm{bc}$ & $1.45 \mathrm{a}$ & $1.56 \mathrm{a}$ & $1.55 \mathrm{a}$ & $1.56 \mathrm{a}$ & 1.48 \\
\hline Vermicomposta & $1.19 \mathrm{c}$ & $1.44 \mathrm{a}$ & $1.55 \mathrm{a}$ & $1.51 \mathrm{a}$ & $1.58 \mathrm{a}$ & 1.45 \\
\hline Inorg.+Orgânica & $1.46 \mathrm{a}$ & $1.45 \mathrm{a}$ & $1.53 \mathrm{a}$ & $1.55 \mathrm{a}$ & $1.63 \mathrm{a}$ & 1.52 \\
\hline
\end{tabular}

"Medias con la misma letras no son significativamente diferentes (Tukey $\mathrm{p} \leq 0.05$ ).

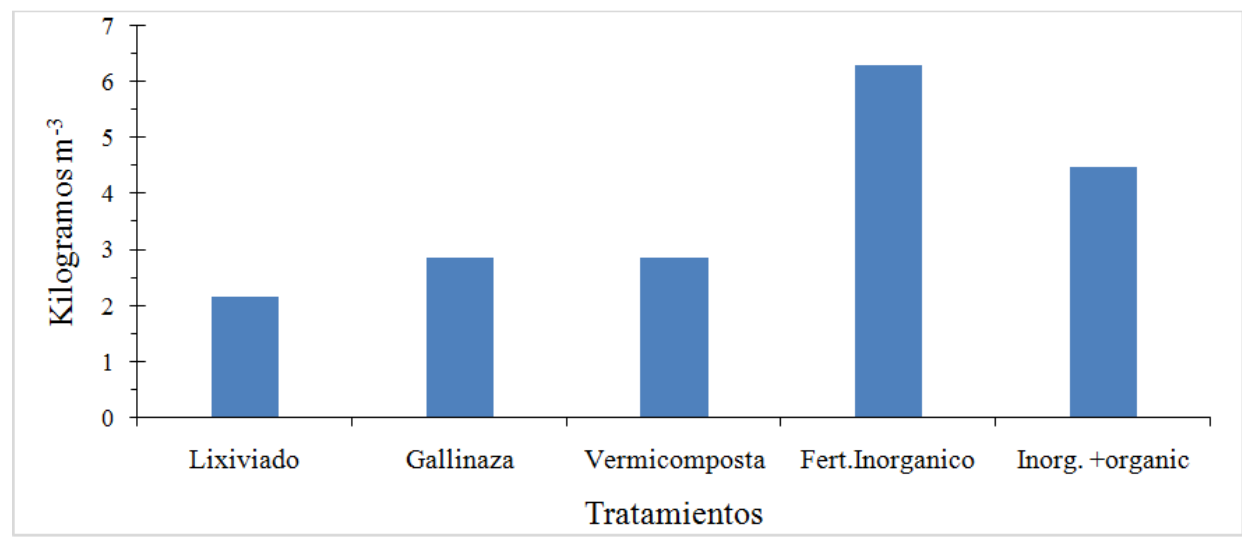

Figura 1. Eficienciadel uso del agua en chile serrano (Capsicum annuum L.), variedad Don Vicente, producido bajo fertilización orgánica e inorgánica encasa sombra

Referente a esto, (Stanhill, 1990) menciona que en la agricultura orgánica el rendimiento se reduce entre 10.0 y $30.0 \%$ respecto a la agricultura convencional.

Longitud: Para la longitud del fruto por planta (Tabla 3) No se observan diferencias significativas en el primer corte, sin embargo, en el segundo corte si hubo diferencias significativas, resultando los mejores tratamientos la fertilización inorgánica y el lixiviado de lombriz con 9.45 y $9.33 \mathrm{~cm}$ respectivamente. Para el tercero, cuarto y quinto corte la tendencia fue la misma y sin diferencias significativas hasta el promedio final, resultando el mejor tratamiento la fertilización inorgánica con $8.84 \mathrm{~cm}$ por fruto, seguido del lixiviado de lombriz con $8.52 \mathrm{~cm}$ por fruto. Los resultados de este trabajo en sentido general están de acuerdos con los obtenidos por Márquez et al. (2013) en el cultivo de chile Piquín en condiciones protegidas quien encontró que la fertilización orgánica incrementa la longitud.

Diámetro: Respecto al diámetro del fruto por planta (Tabla 4) se puede observar que en el primer corte los tratamientos inorgánicos destacaron, siendo el tratamiento inorgánico más extracto de algas marinas el más alto con $1.46 \mathrm{~cm}$, seguido de 1.39 con la fertilización inorgánica habiendo diferencias significativas. A partir del segundo y hasta el quinto corte, no se encontraron diferencias significativas, resultando de igual manera con un mayor diámetro en el promedio final los tratamientos inorgánicos, con $1.83 \mathrm{~cm}$ con fertilización inorgánica y $1.52 \mathrm{~cm}$ con la combinación de algas + fertilización inorgánica.

Eficiencia del Uso del Agua: En cuanto al volumen de agua aplicada al cultivo, se aplicó una lámina total de riego de $61.5 \mathrm{~cm}$, obteniéndose una eficiencia en el uso del agua para el tratamiento fertilización inorgánica de $6.30 \mathrm{~kg} \mathrm{~m}^{-3}$, mientras que para el tratamiento Inorgánico +Algas se obtuvo $4.48 \mathrm{~kg} \mathrm{~m}^{-3}$, siguiendo los tratamientos Gallinaza, Vermicomposta y Lixiviado con 2.85, 2.85 y $2.14 \mathrm{~kg} \mathrm{~m}^{-3}$, respectivamente (Figura 1). Los resultados contrastan con los obtenidos por Inzunza et al. (2007) Ertek et al., (2007) quienesaplicando una lámina de riego de $68.5 \mathrm{~cm}$ obtuvieron $4.2 \mathrm{~kg}$ $\mathrm{m}^{-3}$ de agua aplicada en chile jalapeño.

\section{CONCLUSIÓN}

Los resultados obtenidos de la presente investigación muestran que la fertilización orgánica no proporciona altos rendimientos comparada con la inorgánica debido a la liberación gradual de nutrientes a la planta. Se puede concluir también, que es posible aplicar una lámina de riego menor a la que aplican los agricultores de la zona, ya que se está aplicando una lámina de aproximadamente $70-75 \mathrm{~cm}$ en trabajos semejantes y en esta investigación se comprobó que se puede producir lo mismo, con menor lamina de riego, mostrando así una mayor eficiencia en el uso del agua.

\section{REFERENCIAS}

De La Cruz-Lázaro, E.; Osorio-Osorio, R.; Martínez-Moreno, E.; Lozano, A. J.; Gómez-Vázquez, A. \& Sánchez-Hernández, R. (2010). Use of composts and vermicomposts for organic production of tomato in greenhouses. Interciencia 35(5): 363368.

Du Jardin, P. 2015. Plant biostimulants: definition, concept, main categories and regulation. Sci Hortic. 196: 3-14.

Ertek, A.; Şensoy, S.; Gedik, I. \& Küçükyumuk, C. (2007) “Irrigation scheduling for green pepper (Capsicum annuum L.) grown in field conditions by using Class-Apan evaporation values". American-Eurasian J. Agric. \& Environ. Sci. 2:349-358.

García, E. 1988. Modificaciones al sistema de clasificación climática de Koppen. Offset Larios, México, D.F.

INFORURAL. (2012). Chile, producción nacional. Disponible en: http://www.inforural.com.mx/spip.php?article7381

Inzunza, I. M. A.; Mendoza, S. F.; Catalán, E. A., Villa, M. M.; Sánchez, I. \& Román, A. (2007). "Productividad del chile jalapeño en condiciones de riego por goteo y acolchado plástico". Rev.Fitotec. Mex. 30:429-436.

Márquez, C.; López, S.; Cano, P. \& Moreno, A. (2013). Fertilización orgánica: una alternativa para la producción de chile Piquín bajo condiciones protegidas. Revista Chapingo Serie Horticultura, 19(3): 279-286.

Montaño, N., Simosa, J. \& Perdomo, A. (2009). Respuesta de tres cultivares de berenjena (Solanum melogena L.) a diferentes combinaciones de fertilizante orgánico y fertilizante químico. Revista UDO Agrícola. 9(4): 807-815.

Nagy, P. T. \& Pintér, T. (2014). Effects of Foliar Biofertilizer Sprays on Nutrient Uptake, Yield and Quality Parameters of Blaufrankish (Vitis vinifera L.) Grapes, Communications in Soil Science and Plant Analysis, DOI: 10.1080/00103624. 2014.989016. 
Nicholls, C. \& Altieri, M. (2006). Manejo de la fertilidad de suelos e insectos plaga: armonizando la salud del suelo y la salud de las plantas en los agroecosistemas. Manejo Integrado de Plagas y Agroecología. 77: 8-16.

Partida-Sandoval, A. A. \& Quezada-Camberos, S. M. (2012). De los nombres del chile y sus variedades principales en tierras nayaritas. Revista Fuente. 4(10): 50-55

Rai, N., Ashiya, P. \& Rathore, D. S. (2014). Comparative Study of the Effect of Chemical Fertilizers and Organic Fertilizers on Eisenia foetida. International Journal of Innovative Research in Science, 3(5): 12991-12998.

Rodríguez-Dimas, N., Cano-Ríos, P., Favela Chávez, E., FigueroaViramontes, U., Álvarez, V., Palomo-Gil, A., MárquezHernández, C. \& Moreno-Reséndez, A. (2007). Vermicomposta como alternativa orgánica en la producción de tomate en invernadero. Revista Chapingo Serie Horticultura. 13(2): 185192.
SAS Institute Inc. (2006). The SAS System for Windows release 9.0., USA.

Stanhill, G. (1990). The comparative productivity of organic agriculture. Agriculture, Ecosystems \& Environment 30(1-2): 126. Doi: 10.1016/0167-8809(90)90179-H

Trewavas, A. (2004). A critical assessment of organic farming-andfood assertions with particular respect to the UK and the potential environmental benefits of no-till agriculture. Crop Protection. 23: 757-781.

Van Oosten M. J.; Pepe, O.; De Pascale, S.; Silletti, S. \& Maggio, A. (2017). The role of biostimulants and bioeffectors as alleviators of abiotic stress in crop plants. Chem Biol Techn Agric. 4:5. 